\title{
Sistema de Información @rchivA de la Junta de Andalucía para la gestión de documentos y archivos
}

The @rchivA information system of the Regional Government of Andalusia for document and archival management

\author{
Emilio Martínez Force (1), Ana Melero Casado (2), Antonio Molina González (3), Ma Isabel Valien- \\ TE FABERO (4)
}

Junta de Andalucía. Consejería de Cultura. Dirección General del Libro, Archivos y Bibliotecas; Secreta-ría General Técnica. C/ Conde de Ibarra, 18, 41071. mariai.valiente@juntadeandalucia.es

\begin{abstract}
Resumen
Se relata y discute la experiencia llevada a cabo en la Comunidad Autónoma de Andalucía para la gestión de los documentos y archivos de gestión autonómica con el desarrollo y aplicación del Sistema de Información @rchivA en el marco del modelo de Administración electrónica de la Junta de Andalucía como herramienta horizontal y transversal para el desarrollo de la actividad archivística en esta administración. Se informa de la estructura tecnológica y funcional del sistema, adaptada a las necesidades de la gestión de los documentos a lo largo de su ciclo vital, y a las de la gestión de los archivos como unidades administrativas prestadoras de servicios. Así mismo, se abordan las principales líneas de gestión del propio proyecto.
\end{abstract}

Palabras clave: Gestión documental. Gestión archivística. Archivos. Herramienta corporativa. Sistemas de información. Junta de Andalucía.

\section{Presentación}

El inicio del desarrollo del Sistema de Información @rchivA se sitúa en el año 2005, como una más de las iniciativas de la Junta de Andalucía en el proceso de configuración de una Administración basada en el uso intensivo de las nuevas tecnologías.

Se concibe como uno de elementos habilitantes del modelo de Administración electrónica de esta Administración Autónoma, que junto con otros como son @ries (registro Integrado de Entrada/Salida), @firma (plataforma de autentificación y firma electrónica), Notific@ (sistema de notificaciones telemáticas), Solicit@ (para la generación y presentación telemática de formularios) Clar@ (plataforma de relación con la ciudadanía), Wand@ (Workflow en la Adminis-

\begin{abstract}
This paper reports about an experience of records management automation taking place at the Regional Government of Andalusia. The aim was making possible the document and archival management of its Autonomic Archives, and consisted in the development and application of the @rchivA Information System. It was developd in the context of the Electronic Administration Scheme of the Regional Government of Andalusia, as an cross-cutting and horizontal application for the development of the archival activity in this administration. This article tries to offer a panoramic vision on the state of the technological structure of the @rchivA Information System, that is adapted to the requirements of document management along each step of the whole life cycle of the records, as well as to the requirements of the archival management in administrative units that provide public services. Thereafter, the main management guidelines of the @rchiva Project are presented.
\end{abstract}

Keywords: Document management. Archival management. Archives. Software management. Corporate software. Information systems. Regional Government of Andalusia.

tración andaluza) Trew@ (motor de tramitación), Pago Telemático (plataforma corporativa de pago y presentación de tributos), o la futura Plataforma de Custodia de documentos electrónicos en la cual se trabaja actualmente, constituyen el entramado tecnológico que soporta y posibilita el desarrollo de la administración electrónica en la Junta de Andalucía.

En este marco el S.I. @rchivA se constituye en la herramienta corporativa para la gestión de los documentos, independientemente de su soporte, así como, de los archivos de titularidad o gestión de la Junta de Andalucía, siendo su ámbito de acción transversal a toda la Administración andaluza y abarcando el ciclo completo de los documentos desde las oficinas hasta el archivo histórico. Si bien existen otras iniciativas encaminadas a aplicar las nuevas tecnologías a 
la gestión documental en el contexto de los archivos de nuestro país (1), es este carácter transversal, su amplio ámbito de aplicación territorial y cualitativo, su carácter de herramienta común para la gestión de los documentos en cualquier soporte y de los centros de archivos de cualquier tipología, así como su apertura a otras administraciones públicas (2) constituye a esta experiencia que aquí con ustedes compartimos en una iniciativa singular.

Con ello, los archivos, independientemente de su tipología (centrales, intermedios, históricos), en tanto que unidades administrativas y prestadoras de servicios a la ciudadanía, orientan el desarrollo de su actividad hacia los objetivos estratégicos para el sector público que fueron marcados por la "i2010: la sociedad de la información y los medios de comunicación al servicio del crecimiento y el empleo. Comunicación de la Comisión, de 1 de junio de 2005, al Consejo, al Parlamento. [COM(2005) 229 final - No publicada en el Diario Oficial]", y que la reciente "Ley 11/2007, de 22 de junio, de acceso electrónico de los ciudadanos a los Servicios Públicos. (BOE número 150 de 23 de junio de 2007)" recoge ya como derechos de los ciudadanos.

La implantación del S.I. @rchivA se reguló por la "ORDEN de 20 de febrero de 2007, conjunta de las Consejerías de Justicia y Administración Pública y de Cultura, por la que se regula la implantación y uso del Sistema de Información para la Gestión de los Archivos de titularidad y/o gestión de la Junta de Andalucía (proyecto @rchivA Andalucía). (BOJA n 71 de 11 de abril de 2007)", siendo su ámbito de aplicación todos los órganos, unidades y archivos de titularidad y/o gestión de la Administración de la Junta de Andalucía, de sus organismos autónomos y empresas públicas. La Consejería de Cultura, en razón de sus competencias, es la responsable de dirección, coordinación y mantenimiento del proyecto.

La citada Orden contempla, así mismo, la implantación del S.I. @rchivA en los archivos de los órganos judiciales radicados en Andalucía, implantación que es coordinada desde la Consejería en la que residen las competencias en materia de Justicia.

Atendiendo a su ámbito de aplicación en los archivos de titularidad y/o gestión de la Junta de Andalucía, el S.I. @rchivA es transversal, integrado y único para la gestión de todos los archivos y documentos independientemente de su soporte, tal como se prevé en el Proyecto de Ley de Documentos, Archivos y Patrimonio Documental, actualmente en tramitación, teniendo entre sus objetivos finales el que la Administra- ción Autónoma pueda para garantizar el ejercicio por parte de la ciudadanía de los derechos de acceso a los registros y documentos, y a la información que estos contienen, sin que se incurra en discriminación de la información por soporte y tecnología.

\section{Estructura tecnológica y funcional del sistema}

\subsection{Estructura tecnológica}

Desde un punto de vista tecnológico, podemos señalar que el Sistema de Información @rchivA está desarrollado siguiendo el modelo vista controlador (MVC) de Java2 Enterprise Edition (J2EE), utilizando una arquitectura en tres capas, la de presentación, la de lógica de negocio de la aplicación, y la de datos, conceptualmente separadas.

El sistema de gestión de base de datos utilizado es Oracle, por su carácter corporativo dentro de la Junta de Andalucía y por sus indudables capacidades técnicas. La capa de acceso a los datos o capa de persistencia nos permite aislar a la capa de negocio de la implementación concreta de la base de datos; en nuestro caso está desarrollada con Torque, aunque en la actualidad se está trabajando en su sustitución por Hibernate (que proporciona una mayor potencia y trazabilidad de las actuaciones).

El entorno de trabajo (framework) Struts permite implementar en JAVA de forma ágil el patrón de arquitectura MVC en Java, construyendo el entramado de clases que realizan las funciones de control y workflow de la aplicación, al tiempo que diseñando las páginas dinámicas (JSP y JSTL) que generan el código HTML final a los usuarios en el servidor, donde se ha optado por una solución que consiste en utilizar Tomcat como contenedor para la aplicación, trabajando conjuntamente con Apache como servidor web.

\subsection{Estructura funcional}

El S.I. @rchivA se estructura en diferentes módulos para atender las necesidades tanto de gestión de los documentos como de los propios archivos. Para facilitar el uso de la herramienta estos módulos se organizan en dos submenús:

- El primero "Configuración del Archivo", engloba los módulos que se utilizan la configuración del archivo en el sistema y que permitirán que estos desarrollen su actividad técnica y administrativa.

- El segundo "Gestión del Archivo", agrupa los módulos que se utilizan en el propio desarro- 
llo de su actividad técnica y administrativa por parte de los archivos

Desde los módulos del primer submenú "Configuración del Archivo", los archivos crean y gestionan las estructuras básicas necesarias para la gestión de los documentos y el archivo: datos de identificación del archivo, perfiles de usuarios, cuadros de clasificación, estructura de las relaciones entre los niveles de agrupación documental, depósitos.

Así mismo aportan y mantienen la información horizontal que precisan para ello:, series documentales, signaturas, usuarios, descriptores, normativa, motivos de rechazos.

La gestión de estas estructuras e información básicas recogen la complejidad de la gestión documental incardinada en la gestión administrativa con aplicación de la metodología archivística.

En el funcionamiento de esta estructura tiene especial relevancia la integración del S.I. @rchivA con:

1. Los sistemas de información existentes en la Administración Autónoma para la tramitación de expedientes (independientemente de su soporte: papel, mixtos o electrónicos), creando un flujo de información entre aquellos y el S.I. @rchivA para facilitar la gestión documental y la cadena de custodia.

2. Las distintas plataformas y elementos habilitantes del modelo de Administración electrónica, ya que el S.I. @rchivA es la herramienta para el desarrollo de la actividad administrativa de los archivos.

3. La Plataforma de Custodia de documentos electrónicos de la Junta de Andalucía, repositorio digital seguro para el almacenamiento de documentos electrónicos (actualmente en desarrollo). El S.I. @rchivA es la herramienta mediante la que se controla y gestiona el archivo y custodia del documento electrónico en el repositorio digital seguro, así como el la gestión de los servicios y tratamientos técnico que estos, al igual que el documento en papel, deben soportar y recibir, eliminando en el acceso a la información que los documentos cualquier discriminación por soporte y tecnología de los mismos.

El mantenimiento y evolución de esta estructura funcional, la atención a sus usuarios (desde la especificidad que la diversidad y distinta tipología de archivos requiere) con respeto a la metodología archivística para garantizar el correcto tratamiento de los documentos a lo largo de su ciclo vital completo, así como los objetivos fina- les de servicio a la ciudadanía y apoyo a la propia Administración, son elementos claves de la gestión del proyecto @rchivA.

\section{Gestión del proyecto @rchivA}

La dirección, coordinación y mantenimiento del proyecto el S.I. @rchivA, dado su extenso ámbito de aplicación y que sirve a una Administración Autónoma con nivel competencial de primer rango, lo cual se materializa en un ingente volumen y diversidad de documentos a gestionar, requiere de una infraestructura propia y del despliegue de varias líneas de actuación por parte de la Consejería de Cultura.

Entre ellas destacamos las siguientes:

1. La gestión de los recursos humanos, técnicos y económicos necesarios para el mantenimiento de la infraestructura requerida para el funcionamiento y desarrollo del Sistema.

2. Mantenimiento y desarrollo del Sistema desde el punto de vista lógico, funcional y físico. Desde esta línea se abordan tanto la resoluciones de incidencias, la evolución y perfeccionamiento del Sistema, como el mantenimiento y gestión del equipamiento físico necesario en el que este se aloja.

3. Atención a los usuarios, cuyo número en la actualidad supera los 2.000 . Este servicio se articula a través del uso de la herramienta corporativa para la gestión de incidencias "Servidesk" desarrollada por la Junta de Andalucía. Así mismo, para la prestación de este servicio al usuario se cuenta con el apoyo del Centro del Servicio al Usuario (CSU) de la Consejería de Cultura que garantiza una atención telefónica al usuario en un horario de 08:00 a 21:00 horas.

4. Planificación y gestión del proceso de implantación en los archivos a los que es de aplicación la citada Orden de 20 de febrero de 2007. En la actualidad el sistema está implantado en todos los Archivos Centrales de Consejería, los Archivos Intermedios e Históricos gestionados por la Junta de Andalucía, continuado el proceso en los Archivos Centrales de las Entidades Instrumentales (Agencias administrativas) y en los de la Administración territorial de la Junta de Andalucía.

5. Planificación y dirección de los procesos de migración de datos al S.I. @rchivA procedentes de aplicaciones o herramientas en uso en los archivos con anterioridad al proceso de implantación. 
6. Formación de usuarios y mantenimiento del Aula de Formación Permanente.

7. Aplicación de las líneas de cooperación entre administraciones en materia de TIC recogidas en los planes de Gobierno de la Comunidad Autónoma de Andalucía y en la legislación vigente en materia de administración electrónica.

8. En esta línea destacamos los trabajos técnicos que se están desarrollando para proceder a la liberación del código del Sistema.

9. Desarrollo de servicios on-line.

10.Desde esta línea de actuación se ha llevado a cabo el desarrollo de "@rchivAWeb", módulo Web del sistema para el acceso, de acuerdo con la legislación vigente en la materia, a la información sobre los documentos custodiados en la red de archivos de titularidad y/o gestión autonómica, independientemente de la tipología de estos (centrales, intermedios o históricos). La conexión de @rchivAWeb con el repositorio digital de documentos de archivos "Baida" permite unir a la información archivística de los documentos de libre consulta, la imagen del documento digitalizado.

\section{Conclusiones}

El desarrollo de este sistema de información en el marco del modelo de Administración electrónica de la Junta de Andalucía como un elemento habilitante de la misma y como herramienta horizontal y transversal para el desarrollo de la actividad archivística en esta Administración, ha permitido que la Comunidad Autónoma de Andalucía cuente con un Sistema común, integral e integrado capaz de ofrecer información sobre los documentos custodiados en todos los archivos de titularidad o gestión de la Junta de Andalucía, contribuyendo con ello a la modernización de los Servicios Públicos de la Junta de Andalucía a través de la implantación de las nuevas tecnologías, a la intensificación de los servicios prestados a la ciudadanía a través de las nuevas tecnologías y la participación de esta Comunidad en las políticas de cooperación entre administraciones en materia de TIC.

\section{Notas}

(1) Entre ellos y por su grado de implantación podemos citar a modo de ejemplo los siguientes: Sistema de información y gestión de Archivos del Gobierno de Asturias (SIGIA); Sistema de gestión electrónica de documentos administrativos de la Generalitat de Catalunya (SIGeDA). Así mismo, señalar que en la misma línea otras administraciones han optado por soluciones comerciales de distinto alcance para el desarrollo de estas activida- des a través de las nuevas tecnologías: Archidoc; Archivo3000; Albalá; etc.

(2) La Junta de Andalucía mediante la firma de los correspondientes convenios ha cedido el uso del S.I.@rchivA a la Junta de Extremadura y al Ministerio de Justicia, encontrándose en tramitación la firma de los otros convenios de cesión con otras administraciones públicas. Así mismo en la actualidad se realizan los trabajos encaminados a la liberación del código del Sistema y a la adaptación del mismo para la elaboración de una versión adaptada a instituciones que gestionan el ciclo completo de los documentos.

(3)http://europa.eu/legislation_summaries/employment_and social policy/job_creation measures/c11328 es.htm. (24-03-2010).

\section{Referencias}

Cermeno Martorell, Lluís; Casellas Serra, Lluís-Esteve; Bello Jiménez, Víctor M. (2009). La norma ISO 15489 y la implantación de un sistema de gestión de documentos: (análogos y electrónicos). Las Palmas de Gran Canaria: Anroart, 2009. ISBN 13: 978-84-92628-27-8

Llansó i Sanjuan, Joaquim; Bello Jiménez, Víctor M. (2009). Gestión de documentos electrónicos: la importancia de los sistemas de gestión de documentos: nociones generales. Las Palmas de Gran Canaria: Anroart, 2009. ISBN 13: 978-84-92628-44-5

Rivero Ortega, Ricardo (2008). El expediente administrativo: de los legajos a los soportes electrónicos. Navarra: Aranzadi, 2008. ISBN 13: 978-84-8355-628-3

Training in Electronic Records Management (2009) $=$ Tutorial para la Gestión de Archivos Electrónicos (2009). http://www.irmt.org/educationTrainMaterials.php (201004-15).

Recibido: 15-04-2010. Revisado: 14-06-2010.

Aceptado: 01-07-2010. 